\title{
A Hybrid Time to Digital Converter based on Digital Delay Locked Loop and Analog Time to Amplitude Converter
}

\author{
Imane Malass, Wilfried Uhring, Jean-Pierre Le Normand, Norbert Dumas, Foudil Dadouche \\ ICube, UMR 7357, University of Strasbourg and CNRS \\ 23, rue du Loess, 67037 Strasbourg, France \\ Email: Wilfried.uhring@unistra.fr
}

\begin{abstract}
This paper presents the simulations and characterizations results of a hybrid Time to Digital Converter (TDC) fabricated in $180 \mathrm{~nm}$ standard CMOS. The design combines the traditional Analog Time to Amplitude Converter (TAC) and Digital TDC techniques to obtain a high adjustable time precision. These approach leads to a 3 bits enhancement of the least significant bit resolution (LSB) for the proposed design. The characterization results showed a time precision of $10 \mathrm{ps}$ with an estimated INL of $5.6 \mathrm{ps}$ rms for a $2.5 \mathrm{~ns}$ reference period clock and a 32 cells delay line loop.
\end{abstract}

Keywords-Delay locked loop (DLL), Time to Digital Converter (TDC), Time to Analog Converter (TAC), Time interval measurement.

\section{INTRODUCTION}

Time to Digital Converters are block circuits used to perform high precision time interval measurements where the time interval is defined by the arrival of two signals, a START signal and a STOP signal. TDCs are widely used in many scientific applications such as Time Correlated Single Photon Counting (TCSPC) [1], Fluorescence Life Time Measurement (FLIM) [2], and Diffuse Optical Tomography (DOT) [3].The simplest TDC can be obtained by connecting a very high frequency clock to a counter to determine the number of periods elapsed between the arrivals of the "Start" signal and the "Stop" signal, this approach is quite simple and allows measurements over a wide dynamic range but the asynchronous nature of the "Start" and "Stop" signals leads to measurement errors. Furthermore, the achieved resolution is limited by the used clock frequency as a result good time resolutions require the use of extremely high frequency rates. To reach better resolutions many TDCs have been designed and depending on the methods they employ they are usually classified into digital TDCs or analog TDCs [4]. Analog TDCs [5] consist of a time to amplitude converter (TAC) followed by a conventional analog to digital converter (ADC). The "Start" and "Stop" signals are used to generate a pulse which is transformed by the analog integrator into a voltage fed to an ADC that transforms it into a digital code. With the VLSI technology progress, digital solutions became more popular due to the many advantages of digital circuits compared to analog circuits, especially with the technology scaling trend that led to area reduction, power consumption and faster devices owing to the reduction in gate delays which

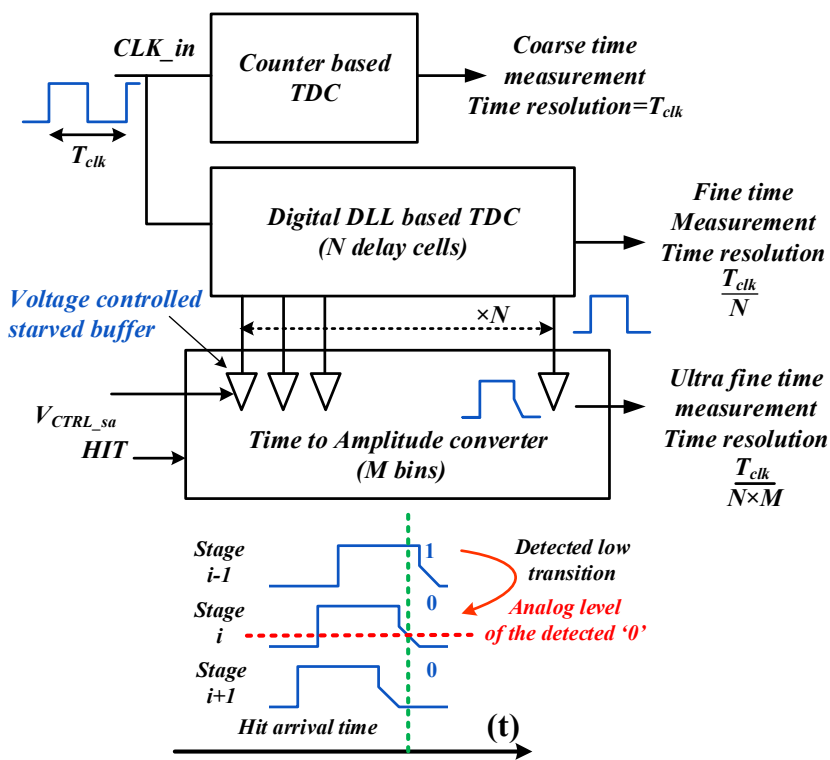

Figure. 1 - The 3 stages conversion scheme

is particularly advantageous to functionalities related to the time domain such as TDCs. Digital TDCs are mainly based on digital delay lines [6], the most straightforward ones measure time intervals by propagating the START signal in a line of buffers which taps are sampled when the STOP signal arrives, the resultant time resolution is equal to the gate delay in the used technology. To achieve sub-gate delay resolutions many methods have been used such as Vernier Delay Lines [7] DLL TDCs [8] and DLL arrays [9]. In general, TACs offer very good resolutions with low power consumption but they suffer from a limited dynamic range. Alternatively, digital DLL-based TDCs offer a reasonable dynamic range but it comes with the price of a more complex design, high power consumption and a large chip area. We recently introduced a hybrid TDC design [10] which makes use of the analog TDC and digital TDC concepts to achieve a high adjustable time resolution that could reach $10 \mathrm{ps}$ and a flexible large dynamic range with a maximum value of $10 \mu \mathrm{s}$. In this paper, we report on the design and characterization of the proposed hybrid TDC implemented in a $180 \mathrm{~nm}$ Standard CMOS technology. 


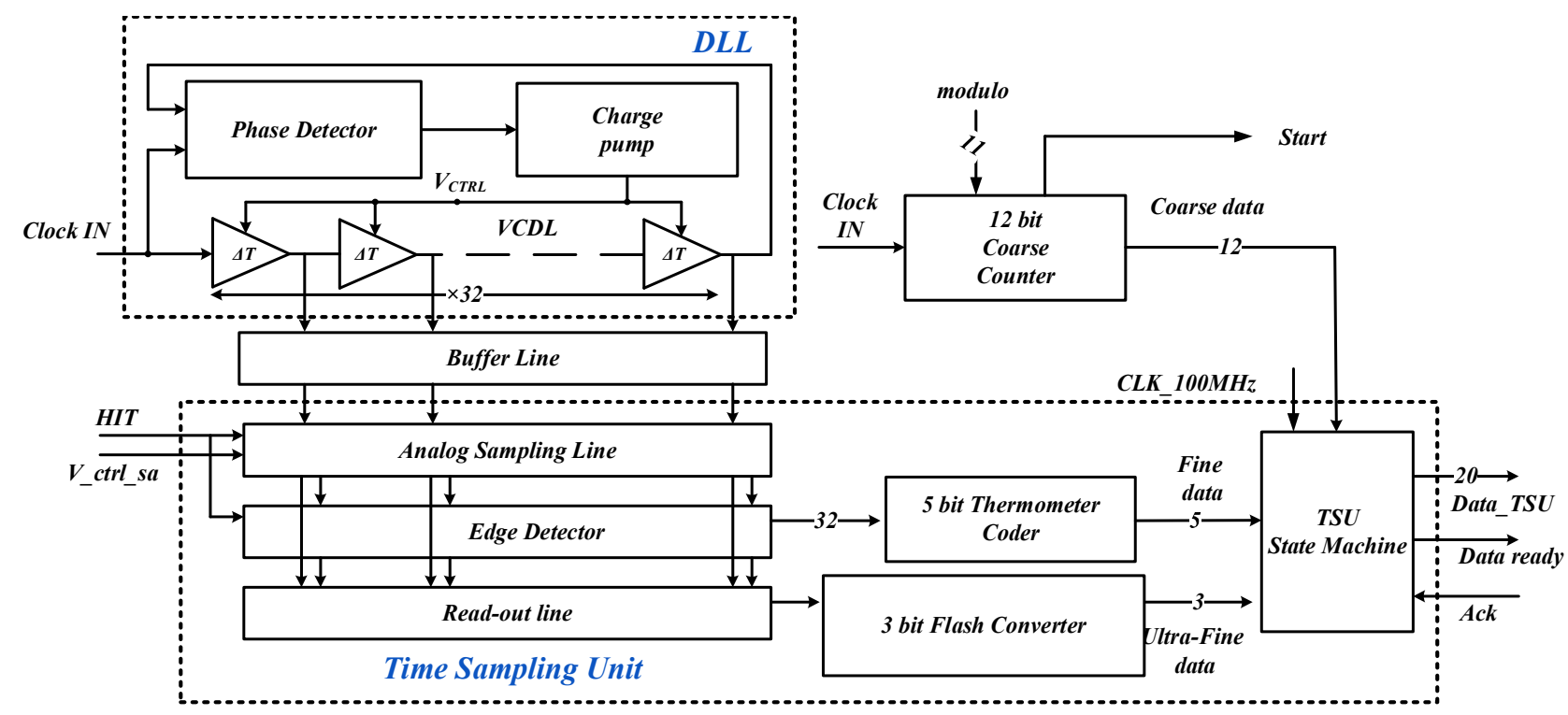

Fig. 2 - Block diagram of the designed hybrid TDC

The paper is organized as follows. In section II we briefly present the hybrid TDC architecture and the time conversion scheme. The characterization results are reported in section III. Finally, we conclude in section IV.

\section{THE HYBRID TDC}

The hybrid TDC can be represented as a 3 stages time converter (Figure.1) where the first stage is a counter-based TDC that performs coarse time measurements with a resolution equal to its clock input's period $(T c l k)$ and offers a high dynamic range. The second stage is a DLL-based TDC that delivers a fine time measurement with a higher resolution of $T_{f}=T c l k / N$ where $N$ is the number of cells in the DLL's voltage controlled delay line (VCDL). The last stage consists of a TAC where the N DLL's taps are used to charge a line of capacitors so that when a HIT signal occurs their output voltage is sampled and held for a fixed duration. The edge slope is then quantized into $M$ equal bins and the stored voltage of the sampling cell where the ' 1 ' to ' 0 ' transition occurred is used to get the ultrafine time measurement resulting in a total time resolution equal to $T_{u f}=T c l k /(M \times N)$. The block diagram of the hybrid TDC is shown in Figure.2, it consists of 3 main parts, a digital 12-bit counter, a DLL with 32 Voltage controlled delay elements and a time sampling unit (TSU). A $400 \mathrm{MHz}$ clock is fed into the coarse counter and the VCDL including 32 delay elements, thus allowing a coarse time of $2.5 \mathrm{~ns}$ and a fine time resolution of 80 ps. The 32 taps of the VCDL are fed into the TSU (Figure.2) which is the central block of the hybrid TDC as it is in charge of carrying the fine and ultrafine time conversions. The TSU was designed to perform several tasks triggered by the arrival of the HIT signal. These tasks include acquiring the coarse time measurement results, sampling the VCDL taps state at the detection moment, determining the VCDL stage where a ' 1 ' to ' 0 ' transition took place in order to acquire the fine time measurement, performing the ultra-fine time conversion based on the analog level in the ' 1 ' to ' 0 ' transition cell to obtain the ultra-fine time measurement and finally transmitting the 20 bits total time conversion data. The TSU includes 4 main parts: 1) The analog sampling Line (ASL) in charge of the sampling and hold task 2) The edge detector to detect the ' 1 ' to ' 0 ' transition stage in the ASL followed by a 5 bit thermometer coder to generate the fine time conversion data 3) The read-out line followed by a 3 bit flash converter to perform the 8 levels ultra-fine time conversion resulting in an ultra-fine time resolution $T_{u f}=10 \mathrm{ps}$ and 4 ) the TSU state machine in charge of collecting the resultant time conversion data and managing the data transmission process.

\section{CHARACTERISATION RESULTS}

The presented hybrid TDC was fabricated in a standard $180 \mathrm{~nm}$ CMOS technology and measurements were conducted to evaluate the fine and ultrafine time conversion operations accomplished by the TSU block of the TDC. Measurement were conducted over a clock operation frequency ranging from $50 \mathrm{MHz}$ to $400 \mathrm{MHz}$ resulting in a time resolution from $80 \mathrm{ps}$ to $10 \mathrm{ps}$. A "Stanford research system DG645" pulse/delay generator was used to perform the measurements. The nominal operation should be to apply a clock signal to Clock_In and to trig the delay generator with the Start Signal generated by the TDC chip. However, the DG645 delay generator is specified with a jitter of less than $25 \mathrm{ps}$ in respect to the signal applied at its input trigger so in order to evaluate the $10 \mathrm{ps}$ resolution of the TDC, this jitter has to be reduced. The trigger jitter of the DG645 can be canceled by operating the delay generator with its own internal trigger and generating both a Clock_In pulse to be propagated through the VCDL and a delayed version of this pulse to be connected to the HIT input of the TSU by two outputs of the delay generator. The residual jitter obtained between these two signals using this setup was evaluated by means of a "LeCroy wave master 13Zi" oscilloscope and it was found to be less than 7 ps rms. Figure. 3 shows the averaged 


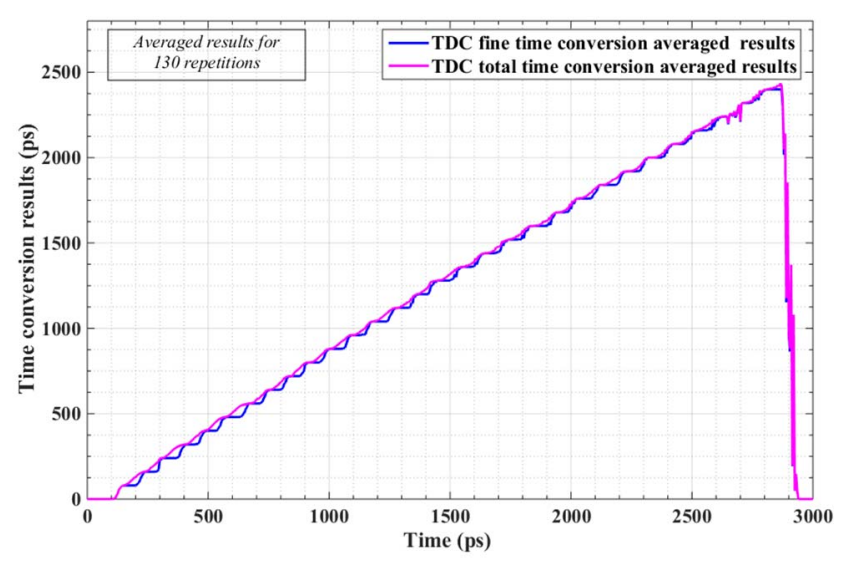

Figure. 3 - Averaged fine time conversion and total time conversion measurements results over a range of 2800 ps for a

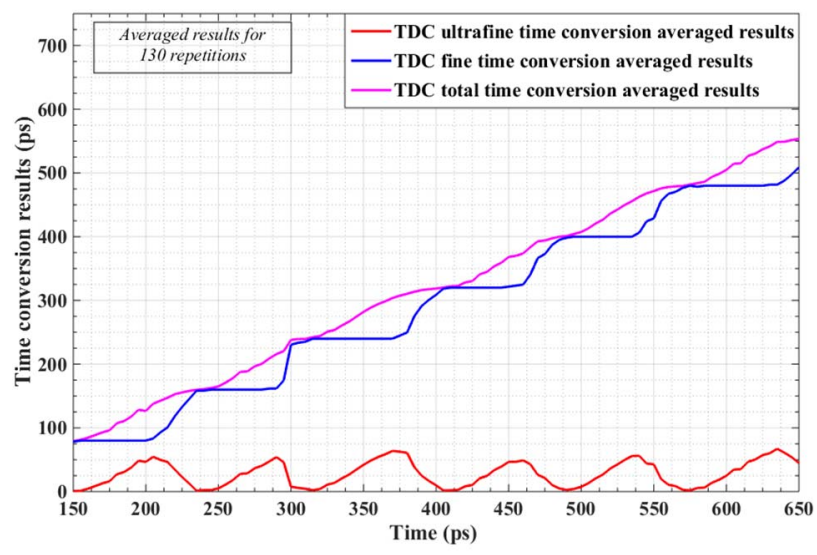

Figure. 4 - Close-up of the averaged measurements results for the ultrafine, fine and total time conversions measurements over a range of $500 \mathrm{ps}$

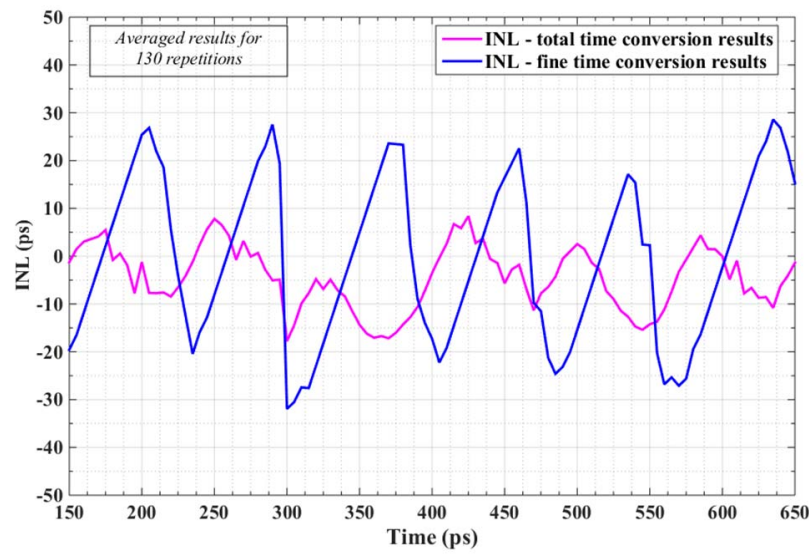

Figure. 5 - Close-up of the estimated INL for the fine and the total time conversions measurements over a range of $500 \mathrm{ps}$

results for 130 measurements of the only fine time conversion and the total time conversion obtained after combining the fine and ultrafine time conversion over a range $2800 \mathrm{ps}$. The stair step artifacts due to the fine resolution quantification is clearly attenuated thank to the ultrafine measurement. A close up view with the fine time conversion results, the ultrafine time conversion results and the total results over a range of $500 \mathrm{ps}$ is scheme is quite noticeable as the INL, shown in Figure.5, shown in Figure. 4. The improvement obtained using the described
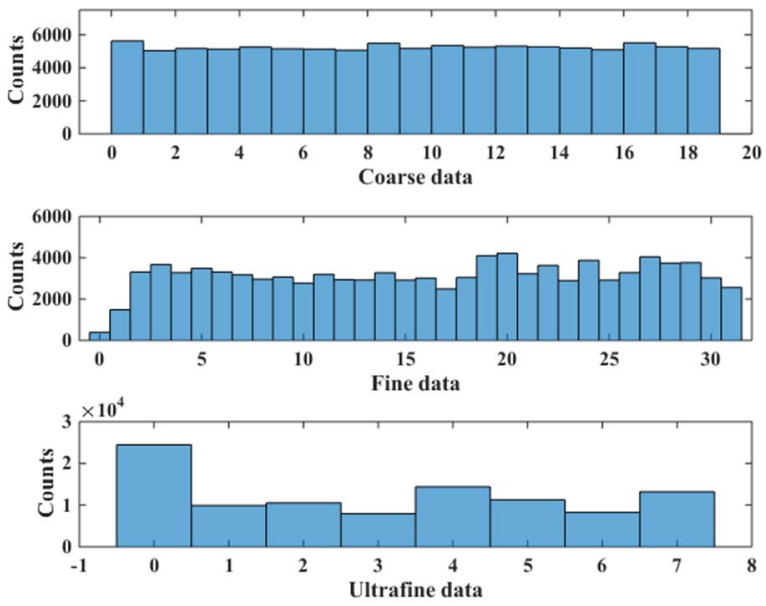

Figure. 6 - Bin density measurement results of the 3 TDC stages for an input clock frequency of $200 \mathrm{MHz}$

is improved from $21 \mathrm{ps}$ rms using the fine time conversion results to $\sim 5.6 \mathrm{ps}$ rms using the complete conversion scheme. The minimal delay step of the Stanford DG645 is 5 ps which is quit high compared to the TDC resolution, thus the measured TDC performance such as the INL are limited by the experimental characterization setup. Figure. 6 shows the bin density measurements results conducted for a clock input frequency of $200 \mathrm{MHz}$ resulting in a time resolution of $20 \mathrm{ps}$. The 3 stages output data are combined to obtain the total data results and the TDC transfer function shown in Figure.7. The coarse counter maximum output was set to 19 hence the limited dynamic range $97.28 \mathrm{~ns}$. These measurements show an irregular bin counts which are due to several factors such as Voltage drop as well as non-linearity resulting from the divergence between real and ideal output of the analog sampling cell. These disparities require careful design and layout consideration to eliminate them as they have direct impact on the TDC reliability. In the case of a targeted application that is based on the technique of time correlated single photon counting, it is possible to compensate the distortion through a compensation algorithm as discussed in [11].

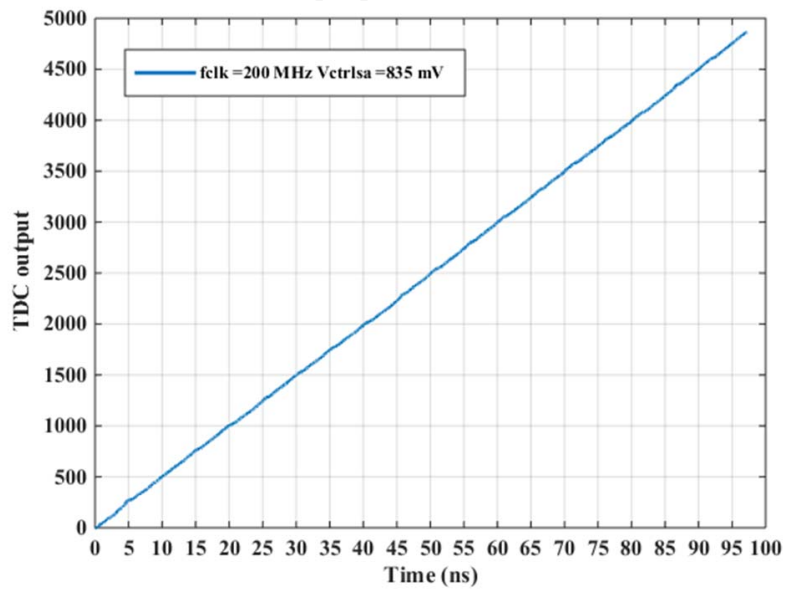

Figure. 7 - TDC transfer function over a dynamic range of $97.28 \mathrm{~ns}$ for an input clock frequency of $200 \mathrm{MHz}$ combining the results of the 3 stages 


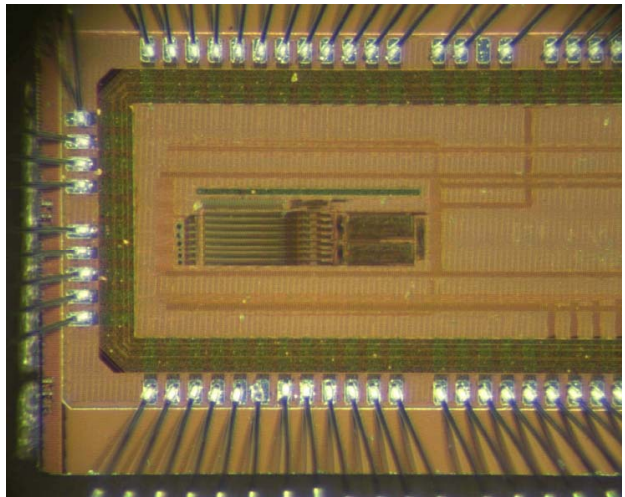

Figure. 8 - Die photo of the TDC array incorporating 8 independent TSUs with common DLL, coarse counter and a Data acquisition unit fabricated in $180 \mathrm{~nm}$ standard CMOS technology

\section{CONCLUSION}

We introduced a hybrid TDC architecture capable of achieving good time resolutions without the need to use extremely high clock frequencies or exceptionally long DLL delay lines, and with reasonable power consumption and silicon area occupation [10]. The hybrid TDC architecture was used to realize a TDC array incorporating 8 independent TSU sharing a DLL, a coarse counter and a data acquisition unit in a $180 \mathrm{~nm}$ standard CMOS technology (Figure. 8). Measurements were performed to test the effectiveness of the proposed time conversion scheme. The hybrid TDC was especially designed to operate with variable input frequencies ranging from $50 \mathrm{MHz}$ to $400 \mathrm{MHz}$ allowing for an achievable time resolution ranging from $80 \mathrm{ps}$ to $10 \mathrm{ps}$. The highest speed operation using a $400 \mathrm{MHz}$ clock input results in a 10 ps time resolution and an estimated INL of $\sim 5.6 \mathrm{ps}$ rms compared to an $80 \mathrm{ps}$ LSB and an INL of 21 ps rms using the DLL based TDC alone. Some irregularities were detected but it was evident that the 3 stages conversion scheme allows for better resolution. Better results should be possible following careful consideration to determine and correct the source of the detected discrepancies.

Table I - Performance Figures of the presented hybrid TDC compared to several state of the art integrated TDCs

\begin{tabular}{|c|c|c|c|c|c|}
\hline & $\begin{array}{c}\text { This } \\
\text { work }\end{array}$ & {$[12]$} & {$[13]$} & {$[14]$} & {$[15]$} \\
\hline Resolution & $10 \mathrm{ps}$ & $97.5 \mathrm{ps}$ & $\begin{array}{c}62.5 \\
\mathrm{ps}\end{array}$ & $5 \mathrm{ps}$ & $17 \mathrm{ps}$ \\
\hline $\begin{array}{c}\text { Dynamic } \\
\text { range }\end{array}$ & $10 \mu \mathrm{s}$ & $256 \mathrm{~ns}$ & $64 \mathrm{~ns}$ & $13 \mathrm{~ns}$ & $\begin{array}{c}160 \\
\mathrm{~ns}\end{array}$ \\
\hline Dead time & $15 \mathrm{~ns}$ & - & - & $150 \mathrm{~ns}$ & 150 \\
\hline DNL & $\begin{array}{c}<0.5 \\
\text { LSB }\end{array}$ & $\begin{array}{c}0.2 \\
\mathrm{LSB}\end{array}$ & $\begin{array}{c}<4 \\
\mathrm{LSB}\end{array}$ & - & - \\
\hline INL & $<5.6 \mathrm{ps}$ & $\begin{array}{c}0.3 \\
\mathrm{LSB}\end{array}$ & $\begin{array}{c}<8 \\
\mathrm{LSB}\end{array}$ & - & $\begin{array}{c}<4 \\
\mathrm{peak}\end{array}$ \\
\hline $\begin{array}{c}\text { Average } \\
\text { power }\end{array}$ & $44 \mathrm{~mW} 1$ & $\begin{array}{c}175 \\
\mathrm{~mW}\end{array}$ & $\begin{array}{c}26.4 \\
\mathrm{~mW}\end{array}$ & $\begin{array}{c}160 \\
\mathrm{~mW}\end{array}$ & $\begin{array}{c}<15 \\
\mathrm{~mW}\end{array}$ \\
\hline $\begin{array}{c}\text { Area } \\
(\mathrm{mm})^{2}\end{array}$ & $0.6 \times 0.36$ & $1.6 \times 1.8$ & - & $1.2 \times 1.1$ & 0.3 \\
\hline
\end{tabular}

\section{REFERENCES}

[1] B. Markovic, A. Tosi, F. Zappa and S. Tisa, "Smart-pixel with SPAD detector and time-to-digital converter for time-correlated single photon counting," IEEE Photonics Society, 2010 23rd Annual Meeting of the, Denver, CO, 2010, pp. 181-182.

[2] R. M. Field, S. Realov and K. L. Shepard, "A 100 fps, Time-Correlated Single-Photon-Counting-Based Fluorescence-Lifetime Imager in $130 \mathrm{~nm}$ CMOS," in IEEE Journal of Solid-State Circuits, vol. 49, no. 4, pp. 867 880, April 2014.

[3] M. Kanoun, L. Arpin, V. P. Rheaume, M. A. Tétreault, Y. BérubéLauzière and R. Fontaine, "A 10-bit, 3 ps rms precision time-to-digital converter for diffuse optical tomography measurements," Electronics, Circuits and Systems (ICECS), 201421 st IEEE International Conference on, Marseille, 2014, pp. 554-557.

[4] Markovic, B.; Tisa, S.; Villa, F.A.; Tosi, A.; Zappa, F., "A HighLinearity, 17 ps Precision Time-to-Digital Converter Based on a SingleStage Vernier Delay Loop Fine Interpolation," Circuits and Systems I: Regular Papers, IEEE Transactions on , vol.60, no.3, pp.557,569, March 2013.

[5] Crotti, M.; Rech, I.; Ghioni, M., "Four Channel, 40 ps Resolution, Fully Integrated Time-to-Amplitude Converter for Time-Resolved Photon Counting," Solid-State Circuits, IEEE Journal of , vol.47, no.3, pp.699,708, March 2012

[6] Minas, N.; Kinniment, D.; Heron, K.; Russell, G., "A High Resolution Flash Time-to-Digital Converter Taking Into Account Process Variability," Asynchronous Circuits and Systems, 2007. ASYNC 2007. 13th IEEE International Symposium on , vol., no., pp.163,174, 12-14 March 2007

[7] Xing, N.; Shin, W.-Y.; Jeong, D.-K.; Kim, S., "High-resolution timetodigital converter utilising fractional difference conversion scheme," Electronics Letters , vol.46, no.6, pp.398,400, March 182010

[8] Mondal, S.A.; Pal, S.; Rahaman, H.; Mondal, P., "Voltage controlled current starved delay cell for Positron Emission Tomography specific DLL based high precision TDC implementation," Computers and Devices for Communication (CODEC), 2012 5th International Conference on , vol., no., pp.1,4, 17-19 Dec. 2012.

[9] Balaji, S.; Srinivasan, K. S., "Low voltage CMOS timing generator using array of digital delay lock loops," Circuits and Systems (MWSCAS), 2012 IEEE 55th International Midwest Symposium on , vol., no., pp.242,245, 5-8 Aug. 2012.

[10] I. Malass, W. Uhring, J. P. L. Normand, N. Dumas and F. Dadouche, "10ps Resolution hybrid time to digital converter in a $0.18 \mu \mathrm{m}$ CMOS technology," New Circuits and Systems Conference (NEWCAS), 2014 IEEE 12th International, Trois-Rivieres, QC, 2014, pp. 105-108

[11] T. Turko, A. Skilitsi , W. Uhring, J-P. Le Normand, N. Dumas, F. Dadouche, J. Léonard, "Time to Digital Converter Transfert Function Improvement using Poisson process event", SIGNAL 2016, Lisbonne, Portugal, pages 50-53, IARIA (Eds.), ISBN: 978-1-61208-48, juin 2016.

[12] B. K. Swann, B.J. Blalock, L. G. Clonts, D. M. Binkley, J. M. Rochelle, E. Breeding, and K. M. Baldwin, "A 100-ps time-resolution CMOS timeto-digital converter for positron emission tomography imaging applications," IEEE Journal of Solid-State Circuits, vol. 39, no. 11, pp. 1839-1852, 2004.

[13] Field, R. M., Realov, S. and Shepard, K. L., "A 100-fps, Time-Correlated Single-PhotonCounting-Based Fluorescence-Lifetime Imager in 130-nm CMOS," IEEE Journal of Solid-State Circuits, vol. 49, no. 4, pp. 867 880, 2014.

[14] M. Kanoun, Y. Berube-Lauziere and R. Fontaine, "High precision timeto-amplitude converter for diffuse optical tomography applications," in Design and Technology of Integrated Systems in Nanoscale Era, 2008. DTIS 2008. 3rd International Conference, Tozeur, 2008.

[15] M. B., T. S., V. F, T. A and Z. F., "A High-Linearity, 17 ps Precision Time-to-Digital Converter Based on a Single-Stage Vernier Delay Loop Fine Interpolation," Circuits and Systems I: Regular Papers, IEEE Transactions, vol. 60, no. 3, pp. 557-569, 2013.

\footnotetext{
${ }^{1}$ with $35 \mathrm{~mW}$ consumed by the DLL alone
} 